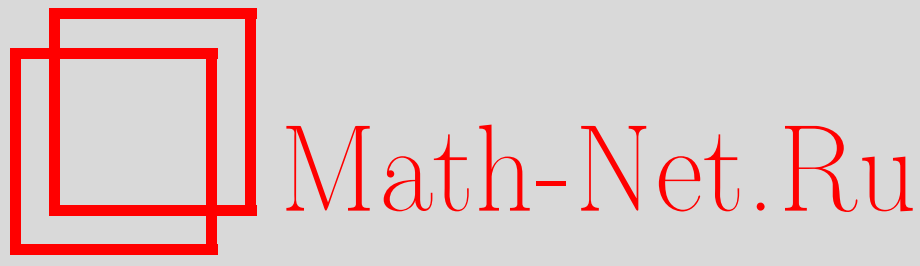

Обшероссийский математический портал

Л. А. Мощевитина, О целых точках в многоугольниках, $M a$ тем. заметки, 1999, том 65, выпуск 4, 629-632

DOI: https://doi.org/10.4213/mzm1092

Использование Общероссийского математического портала Math-Net.Ru подразумевает, что вы прочитали и согласны с пользовательским соглашением http://www . mathnet.ru/rus/agreement

Параметры загрузки:

IP: 18.234 .197 .8

26 апреля 2023 г., 11:05:45 


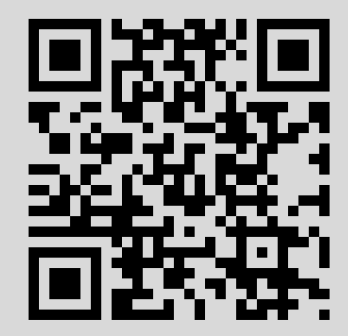




\section{О ЦЕЛЫХ ТОЧКАХ В МНОГОУГОЛЬНИКАХ}

\section{Л. А. Мощевитина}

1. Введение. Работа посвящена вопросу о распределении целых точек в многоугольниках. Пусть $P$ - произвольный $s$-угольник на плоскости $\mathbb{R}^{2}, P+x$ - его сдвиг на вектор $x \in \mathbb{R}^{2}, t P-$ его образ при гомотетии относительно начала координат с коэффициентом $t, S(P)$ - его площадь. Определим

$$
R(P)=\sup _{x \in \mathbb{R}^{2}}\left|\operatorname{card}\left((P+x) \cap \mathbb{Z}^{2}\right)-S(P)\right|
$$

Представляет интерес асимптотическое поведение $R(t P)$ при $t \rightarrow \infty$. Хорошо известно [1]-[3], что для иррациональных многоугольников (т.е. таких, что угловые коэффициенты $\alpha_{j}, j=1, \ldots, s$, прямых линий, задающих стороны, иррациональны) $R(t P)=o(t)$. Для многоугольников, у которых неполные частные разложения в цепную дробь угловых коэффициентов $\alpha_{j}, j=1, \ldots, s$, ограничены в совокупности, известна (см., например, [3], [4]) оценка $R(t P) \ll \log t$, причем константа в знаке “«” зависит только от $s$ и максимума неполных частных для угловых коэффициентов. В общем случае этот результат неулучшаем. Специальный случай, когда $P$ является параллелограммом и неполные частные обоих угловых коэффициентов $\alpha_{1}, \alpha_{2}$ ограничены, рассматривался в [5], где получено уточнение оценки $R(t P) \ll \log t$ для некоторой последовательности значений $t_{\nu} \rightarrow \infty$.

Мы получим улучшение общего результата $R(t P)=o(t)$ для многоугольников с рационально зависимыми угловыми коэффициентами сторон. Возможность улучшения основана на качественном анализе двумерных совместных приближений Дирихле.

2. Уточнение теоремы Дирихле. Хорошо известная теорема Дирихле [6] в двумерном случае утверждает, что для двух вещественньх чисел $\alpha_{1}$ и $\alpha_{2}$, по крайней мере одно из которых иррационально, найдется бесконечно много целочисленных векторов $\left(p, a_{1}, a_{2}\right)$ таких, что

$$
\left|\alpha_{j} p-a_{j}\right|<p^{-1 / 2}, \quad j=1,2, \quad \text { НОД }\left(p, a_{1}, a_{2}\right)=1 .
$$

Мы уточним эту теорему на основе к лассического алгорифма вытягивания параллелепипедов (цепи Минковского-Вороного [7]-[9]). Сформулируем основной результат. 
Теорема 1. Пусть действительные числа $\alpha_{1}, \alpha_{2}$ таковы, что их отношение $\alpha_{1} / \alpha_{2}$ иррачионально. Тогда найдется бесконечная последовательность челочисленных векторов $\left(q_{\nu}, a_{1, \nu}, a_{2, \nu}\right)$ таких, что

$$
\begin{gathered}
\left|\alpha_{j} q_{\nu}-a_{j, \nu}\right|<q_{\nu}^{-1 / 2}, \quad j=1,2, \\
\text { НОД }\left(q_{\nu}, a_{1, \nu}, a_{2, \nu}\right)=1, \\
\text { НОД }\left(a_{1, \nu}, a_{2, \nu}\right)=d_{\nu} \ll q_{\nu}^{1 / 2}, \quad q \rightarrow \infty .
\end{gathered}
$$

Теорема 1 анонсирована автором на конференции [10].

ДокАзАтЕльство. В трехмерном пространстве $\mathbb{R}^{3}$, отнесенном к координатам $\left(x, y_{1}, y_{2}\right)$, определим параллелепипед $\Pi(q, \sigma)$, задаваемый соотношением

$$
\Pi(q, \sigma)=\left\{\left(x, y_{1}, y_{2}\right) \in \mathbb{R}^{3}: 0<x<q,\left|\alpha_{j} x-y_{j}\right|<\sigma\right\}, \quad \sigma<\frac{1}{2} .
$$

Опишем алгоритмически процесс построения цепи параллелепипедов. Выберем параллелепипед $\Pi_{1}$ указанного вида такой, что в нем имеется ровно одна нетривиальная целая точка, причем она лежит на ребре грани $\left\{x=q_{1}\right\}$ параллелепипеда $\Pi_{1}$. Обозначим эту точку $\zeta_{1}=\left(q_{1}, a_{1,1}, a_{2,1}\right)$. Дальнейшее построение проведем индуктивным образом. Пусть уже построены параллелепипеды $\Pi_{\mu}=\Pi\left(q_{\mu}, \sigma_{\mu}\right), \mu=1, \ldots, \nu$, с нетривиальными цельми точками $\zeta_{\mu}=\left(q_{\mu}, a_{1, \mu}, a_{2, \mu}\right)$, $\mu=1, \ldots, \nu$, лежащими на ребрах граней $\left\{x=q_{\mu}\right\}$ соответствующих параллелепипедов, причем

$$
q_{1}<q_{2}<\cdots<q_{\nu}, \quad \frac{1}{2}>\sigma_{1} \geqslant \sigma_{2} \geqslant \cdots \geqslant \sigma_{\nu}, \quad \Pi_{\mu} \cap \mathbb{Z}^{3}=\left\{0, \zeta_{\mu}\right\}, \quad \mu=1, \ldots, \nu .
$$

Построим параллелепипед $\Pi_{\nu+1}$. Будем рассматривать все параллелепипеды вида $\Pi\left(q, \sigma_{\nu}\right)$, где $q>q_{\nu}$. Определим $q_{\nu+1}$ как минимальное натуральное число такое, что $\Pi\left(q_{\nu+1}, \sigma_{\nu}\right)$ содержит кроме $\zeta_{\nu}$ еще ровно одну нетривиальную целую точку $\zeta_{\nu+1}=\left(q_{\nu+1}, a_{1, \nu+1}, a_{2, \nu+1}\right)$. Положим

$$
\sigma_{\nu+1}=\max _{j=1,2}\left|\alpha_{j} q_{\nu+1}-a_{j, \nu+1}\right|, \quad \Pi_{\nu}^{*}=\Pi\left(q_{\nu+1}, \sigma_{\nu}\right), \quad \Pi_{\nu+1}=\Pi\left(q_{\nu+1}, \sigma_{\nu+1}\right) .
$$

Цепь построена.

Отметим, что строго внутри вспомогательного параллелепипеда $\Pi_{\nu}^{*}$ целых точек нет и, следовательно, применяя теорему Минковского о выпуклом теле, получаем неравенство $q_{\nu+1} \sigma_{\nu}^{2} \leqslant 1$.

Лемма 1. Существует бесконечно много пар точек $\zeta_{\nu}, \zeta_{\nu+1}$, которые при ортогональной проекции на координатную плоскость $\left(y_{1}, y_{2}\right)$ не проецируются на одну прямую, проходящ,ую через начало координат 0.

ДоКАЗАТЕЛЬСТВо. Предположим,что это не так, т.е. все точки $\zeta_{\nu}$, начиная с некоторого номера $\nu_{0}$, проецируются на одну прямую $m$, причем $0 \in m$. Это означает, что все точки $\zeta_{\nu}, \nu=\nu_{0}$, $\nu_{0}+1, \ldots$, и 0 лежат в одной плоскости $\pi$ перпендикулярной координатной плоскости $0 y_{1} y_{2}$. Очевидно, что общая ось параллелепипедов $\Pi_{\nu}$, направляющий вектор которой имеет координаты $\left(1, \alpha_{1}, \alpha_{2}\right)$, также лежит в плоскости $\pi$. Но тогда вектор $\left(1, \alpha_{1}, \alpha_{2}\right)$ проецируется на прямую $m$ и, таким образом, $a_{1, j} / a_{2, j}=\alpha_{1} / \alpha_{2}, j=\nu_{0}, \nu_{0}+1, \ldots$ Последнее противоречит иррациональности отношения $\alpha_{1} / \alpha_{2}$.

Лемма доказана.

ЛЕмма 2. Пусть точки $\zeta_{\nu} u \zeta_{\nu+1}$ не проечируются на одну прямую, проходящую через начало координат 0 . Тогда выполнено неравенство

$$
d_{\nu+1} \ll q_{\nu+1}^{1 / 2} .
$$


ДоКАЗАТЕльСтво. Рассмотрим проекцию $K$ вспомогательного параллелепипеда $\Pi_{\nu}^{*}$ на координатную плоскость $0 y_{1} y_{2}$. Она представляет собой объединение всех квадратов со сторонами длины $2 \sigma_{\nu}$ параллельными осям координат и с центрами на отрезке, соединяющем точки $(0,0)$ и $\left(q_{\nu+1} \alpha_{1}, q_{\nu+1} \alpha_{2}\right)$. Очевидно, что для площади $S(K)$ проекции рассматриваемого параллелепипеда выполняется неравенство

$$
S(K) \ll \sigma_{\nu} q_{\nu+1} .
$$

Для получения оценки снизу для $S(K)$ рассмотрим треугольник $T$ с вершинами $(0,0),\left(a_{1, \nu}, a_{2, \nu}\right)$, $\left(a_{1, \nu+1}, a_{2, \nu+1}\right)$, содержашийся в $K$. На стороне треугольника $T$, соединяющей вершины $(0,0)$ и $\left(a_{1, \nu+1}, a_{2, \nu+1}\right)$, лежат, кроме нуля, ровно $d_{\nu+1}$ целых точек. Следовательно, для его площади $S(T)$ выполняется неравенство

$$
\frac{d_{\nu+1}}{2}<S(T)<S(K)
$$

Объединяя (5), (6) и замечание, получаем (4).

Лемма доказана.

Из изложенного вьше сразу вытекает утверждение теоремы. Соотношения (1), (2) выполняются в силу принадлежности точки $\zeta_{\nu}$ грани $\left\{x=q_{\nu}\right\}$ параллелепипеда $\Pi_{\nu}$. Соотношение (3) обеспечивается выбором точки $\zeta_{\nu+1}$ (лемма 1$)$, для которой согласно лемме 2 выполняется неравенство (4).

3. Целые точки в многоугольниках. Будем говорить, что $2 s$ чисел $\alpha_{1, j}, \alpha_{2, j}$ обладают $D$-свойством, если существует бесконечно много примитивных целочисленных наборов $p, a_{1, j}$, $a_{2, j}, j=1, \ldots, s$, таких, что

$$
\begin{gathered}
\left|p \alpha_{1, j}-a_{i, j}\right|<C_{1}(\alpha) p^{-\rho}, \quad \rho>0, \\
\operatorname{НОД~}\left(a_{1, j}, a_{2, j}\right)=d_{j}<C_{2}(\alpha) p^{\gamma}, \quad \gamma<1 .
\end{gathered}
$$

ПРЕДЛОЖЕНИЕ. Пусть $s$-угольник $P$ таков, что для координат векторов $\left(\alpha_{1, j}, \alpha_{2, j}\right)$, $j=1, \ldots, s$, его сторон выполнено $D$-свойство с некоторыми $\rho, \gamma$. Тогда существует последовательность $t_{\nu} \rightarrow \infty$ такая, что $R(t P) \ll t^{1-\varkappa}$ с $\varkappa=\min \{\rho, 1-\gamma\} \in(0,1)$.

ДоКАЗАТЕльСТво основано на следующей известной лемме (см. [11], [3]).

Лемма 3. Пусть $\alpha_{j}=\alpha_{1, j} / \alpha_{2, j}, j=1, \ldots, s$. Тогда выполняется неравенство

$$
|R(t P)| \ll \sum_{j=1}^{s}\left|\sum_{n}\left(\left\{\alpha_{j} n+\delta_{j}\right\}-\frac{1}{2}\right)\right|+O(k),
$$

где $\delta_{j}$ - некоторые действительные числа, $k$ - число челых точек на граниче $t P$ и суммирование ведется в пределах $z_{j}<n \leqslant t \alpha_{1, j}+z_{j}$, причем $z_{j}-$ координата проекции $j$-й вершины многоугольника $t P$ на ось $y_{1}$.

Предложение вытекает из леммы 3 , если положить $t=p$ для некоторого $p$ из $D$-свойства. Иррациональность отношения $\alpha_{j}$ обеспечивает то, что $k=O(s)$. Каждая сумма

$$
\sum_{n}\left(\left\{\alpha_{j} n+\delta_{j}\right\}-\frac{1}{2}\right)
$$

приводится к виду

$$
\sum_{n}\left(\left\{\frac{n}{a_{1, j}^{*}}+O\left(p^{-\varkappa}\right)\right\}-\frac{1}{2}\right),
$$

где $a_{1, j}^{*}=a_{1, j} / d_{j}$. Если $0<n / a_{1, j}^{*}+O\left(p^{-\varkappa}\right)<1 / 2$, то знак дробной доли $\{\cdot\}$ можно отбросить, а сумма по остальным $n$ дает вклад $d O\left(p^{1-\varkappa} / d\right)=O\left(p^{1-\varkappa}\right)$. В итоге $|R(t P)|=O\left(p^{1-\varkappa}+p^{\gamma}\right)$, и предложение доказано.

Назовем две пары вещественных чисел $\left(\alpha_{1}, \alpha_{2}\right)$ и $\left(\beta_{1}, \beta_{2}\right)$ рачионально зависимыми, если $\alpha_{1}=\lambda_{1} \beta_{1}, \alpha_{2}=\lambda_{2} \beta_{2}, \lambda_{1}, \lambda_{2} \in \mathbb{Q}$.

Из теоремы 1 вытекает, что если все векторы $\left(\alpha_{1, j}, \alpha_{2, j}\right)$ сторон многоугольника $P$ попарно рационально зависимы, то $D$-свойство выполнено с $\gamma=1 / 2, \rho=1 / 2$. Таким образом, имеет место 
Теорема 2. Пусть векторы сторон многоугольника $P$ рационально зависимы (в определенном више смисле) $и \alpha_{1,1} / \alpha_{1,2} \notin \mathbb{Q}$. Тогда для некоторой последовательности $t_{\nu} \rightarrow+\infty$ виполнено $R\left(t_{\nu} P\right) \ll t_{\nu}^{1 / 2}$.

\section{СПИСОК ЦИТИРОВАННОЙ ЛИТЕРАТУРЫ}

1. Hardy G. H., Littlewood J. E. // Abh. Math. Sem. Univ. Hamburg. 1922. V. 1. P. 212-249. 2. Khintchine A. // Math. Z. 1923. V. 18. №3-4. P. 289-306. 3. Скриганов М. М. // Алгебра и анализ. 1989. Т. 16. №2. С. 207-228. 4. Skriganov M. // Ann. Inst. Fourier (Grenoble). 1993. V. 43. № 2. Р. 313-323. 5. Рузимурадов X. X. // Аналитическая теория чисел и теория функций. Вып. 11. Зап. научных семин. ПОМИ. 1993. Т. 204. С. 82-89. 6. Касселс Дж. В. С. Введение в теорию диофантовых приближений. М.: ИЛ, 1961. 7. Minkovski H. // Ann. Sci. École Norm. Sup. (3). 1896. V. 13. Р. 41-60. 8. Вороной Г. Ф. // Избранные труды. Киев: Изд-во АН УССР, 1952. С. 197-391. 9. Делоне Б. Н. Петербургская школа теории чисел. М.-Л.: Изд-во АН СССР, 1947. 10. Мощевитина Л. А. // Конференция "Современные проблемы теории чисел и ее приложения" (Тула 9-14 сентября, 1996). Тезисы докл. Тула, 1996. С. 103. 11. Виноградов И. М. Основы теории чисел. М.: Наука, 1981.

Московский государственный университет им. М.В. Ломоносова 